\title{
Local Pooling Factor of Multihop Wireless Networks
}

\author{
Peng-Jun Wan*, Minming $\mathrm{Li}^{\dagger}$, Lixin Wang ${ }^{\ddagger}$, Zhu Wang* and Ophir Frieder ${ }^{\S}$ \\ * Department of Computer Science, Illinois Institute of Technology \\ Emails:wan@cs.iit.edu, zwang59@iit.edu \\ $\dagger$ Department of Computer Science, City University of Hong Kong \\ Email: minmli@cs.cityu.edu.hk \\ $\ddagger$ Department of Math and Computer Science, Paine College \\ Email: lwang@paine.edu \\ $\S$ Department of Computer Science, Georgetown University \\ Email: ophir@ir.cs.georgetown.edu
}

\begin{abstract}
Longest Queue First (LQF) is a well-known link scheduling strategy in multihop wireless networks. Its throughput efficiency ratio was shown to be exactly the local pooling factor (LPF) of the multihop wireless network in a recent seminar work by Joo et al. [5]. Under the 802.11 interference model with uniform interference radii, the LPF of a multihop wireless network was known to be at least $1 / 6$. However, little is known about the LPF of a multihop wireless network under the 802.11 interference model with arbitrary interference radii or under the protocol interference model. In this paper, we derive constant lower bounds on LPFs of these multihop wireless networks. Specifically, under the 802.11 interference model with arbitrary interference radii, the $\mathbf{L P F}$ is at least $1 / 16$. Under the protocol interference model, if the communication radius of each node is at most $c$ times its interference radius for some $c<1$, then the LPF is at least $1 /\left(2\left(\left\lceil\pi / \arcsin \frac{1-c}{2}\right\rceil-1\right)\right)$.

Index Terms-Link scheduling, interference, longest queue first, throughput efficiency ratio, local pooling factor.
\end{abstract}

\section{INTRODUCTION}

Link scheduling is critical to achieving good network throughput in multihop wireless networks. The seminal work of Tassiulas and Ephremides [10] provided a throughputoptimal scheduler, which can schedule all attainable traffic flows without resulting an unbounded queue. However, this optimal scheduler has exponential running time in general. Thus various suboptimal scheduling strategies that trade-off throughput for simplicity have been proposed in [1], [2], [4], [5], [8], [9], [13], [14], [15] amongst others. Among them, the strategy Longest Queue First (LQF), also termed Greedy Maximal Scheduling (GMS) [5], achieves the best throughput efficiency ratio while still enjoying the implementation simplicity. It selects in the first-fit manner a maximal independent set of links in the decreasing order of their queue lengths. At each step, the link with longest queue is selected and then this selected link and the links with which it interferes are removed from the list of candidate links. The selection terminates when there are no more candidate links.

Recently, Joo et al. [5] made a remarkable contribution to fully characterizing the throughput efficiency ratio of LQF. Built upon the prior works by Dimakis and Walrand [3] which presented sufficient conditions for LQF to achieve $100 \%$ throughput, they proved that the throughput efficiency ratio of LQF is exactly the local pooling factor (LPF) of the conflict graph of the communication links. The LPF is a pure graphtheoretic parameter. Thus, the works by Joo et al. [5] built an elegant bridge between a queuing-theoretic parameter and a graph-theoretic parameter. Under the 802.11 interference model with uniform interference radii, the LPF is shown to be at least $1 / 6$ in [5]. Sparked by the works in [5], Leconte el al. [6] and Li el al. [7] presented some properties of LPF. Leconte el al. [6] derived tighter lower bounds on LPF in networks of size at most 28 under the 802.11 interference model with uniform interference radii. Li el al. [7] gave an alternative definition of LPF and also introduced a refined notion of LPF. All of these bounds on the LPF are restricted to the 802.11 interference model with uniform interference radii. Little is known about the LPF, and accordingly the throughput efficiency ratio of $\mathbf{L Q F}$, under other interference models.

The main objective of this paper is to obtain tight lower bounds on LPF under the 802.11 interference model with arbitrary interference radii or the protocol interference model with arbitrary interference radii. Either of these general interference models lacks the rich physical nature of the wireless interference possessed by the 802.11 interference model with uniform interference radii. As the result, the bounding techniques developed in the early works [5] [6] [7] can hardly be employed to derive any tight lower bounds on LPFs under these general interference models. Therefore, we were pressed to develop some new powerful techniques for bounding LPFs under these general interference models. For this purpose, we first conduct a general graph-theoretic study of the LPF of an arbitrary graph in Section II. We discover an intrinsic relation between the LPF and a graph-theoretic parameter called inward local independence number (ILIN): For any graph, its LPF is no less than one half of the multiplicative inverse of its ILIN. Therefore, under the 802.11 interference model or the protocol interference model, any upper bound on the ILIN of the conflict graph of communication links can immediately lead to tight lower bounds on the LPF. In Section 
III, we derive the tight upper bounds on the ILIN in multihop wireless networks under the 802.11 interference model or the protocol interference model. With these upper bounds, we are able to establish the following two lower bounds on the LPF:

- Under the 802.11 interference model with arbitrary interference radii, the LPF is at least $1 / 16$.

- Under the protocol interference model, if the communication radius of each node is at most $c$ times its interference radius for some $c<1$, then the LPF is at least $1 /\left(2\left(\left\lceil\pi / \arcsin \frac{1-c}{2}\right\rceil-1\right)\right)$.

The following standard notations and terms are adopted throughout this paper. Let $S$ be a finite subset. For any real function $f \in \mathbb{R}^{S}$ and any subset $S^{\prime} \subseteq S, f\left(S^{\prime}\right)$ denotes $\sum_{e \in S^{\prime}} f(e)$. Let $G=(V, E)$ be an undirected graph with vertex set $V$. For any subset $U$ of $V, G[U]$ denotes the subgraph of $G$ induced by $U$. For any node $v \in V, N_{G}(v)$ denotes the set of neighbors of $v$ in $G$, and $N_{G}[v]$ denotes $\{v\} \cup N_{G}(v)$. Suppose that $H$ is digraph with vertex set $V$. For any subset $U$ of $V, H[U]$ denotes the subgraph of $H$ induced by $U$. For each $v \in V, N_{H}^{i n}(v)$ denotes the set of in-neighbors of $v$ in $H$, and $N_{H}^{i n}[v]$ denotes $\{v\} \cup N_{H}^{i n}(v)$; similarly, $N_{H}^{\text {out }}(v)$ denotes the set of out-neighbors of $v$ in $H$, and $N_{H}^{\text {out }}[v]$ denotes $\{v\} \cup N_{H}^{\text {out }}(v)$. The Euclidean distance between two points $u$ and $v$ in the plane is denoted by $\|u v\|$.

\section{LPF OF GRAPHS}

In this section, we present a general graph-theoretic treatment of the local pooling factors of arbitrary graphs. Let $G=(V, E)$ be an undirected graph. A subset $I$ of $V$ is an independent set (IS) of $G$ if no two nodes in $I$ are adjacent. If $I$ is a independent set of $G$ but no proper superset of $I$ is a independent set of $G$, then $I$ is called a maximal independent set of $G$. An independent set of the largest size is called a maximum independent set. Let $\mathcal{I}_{G}$ be the collection of all independent sets of $G$, and $\mathcal{I}_{G}^{*}$ be the collection of all maximal independent sets of $G$. Denote by $\Omega_{G}^{*}$ the convex hull of the incident vectors of sets in $\mathcal{I}_{G}^{*}$. Then, $\Omega_{G}^{*}$ is a polytope in the $|V|$-dimensional spaces, and is referred to as the maximal independence polytope of $G$. Define

$$
\sigma_{G}=\min \left\{c \in \mathbb{R}^{+}: \exists x, y \in \Omega_{G}^{*} \text { s.t. } x \leq c y\right\} .
$$

Clearly, $0<\sigma_{G} \leq 1$. The local pooling factor (LPF) of $G$ is

$$
\sigma_{G}^{*}=\min _{\emptyset \neq U \subseteq V} \sigma_{G[U]}
$$

We will derive two lower bounds on the LPF of $G$ in the two subsections respectively.

\section{A. The First Lower Bound}

In this subsection, we present a lower bound on LPF in terms of a graph-theoretic parameter termed as the inward local independence number. An orientation of $G$ is a digraph obtained from $G$ by imposing an orientation on each edge of $G$. Suppose that $H$ is an orientation of $G$. The inward local independence number (ILIN) of a node $v$ in $H$ is defined to be

$$
\alpha_{H}^{i n}(v)=\max \left\{|I|: I \subseteq N_{H}^{i n}[v], I \in \mathcal{I}\right\}
$$

The inward local independence number (ILIN) of $H$ is defined to be

$$
\beta_{H}=\max _{v \in V} \alpha_{H}^{i n}(v)
$$

The inward local independence number (ILIN) of $G$, denoted by $\beta_{G}^{*}$, is defined as the smallest ILIN of all possible orientations of $G$. The theorem below gives a relation between the LPF and the ILIN of $G$, which provides a means to lower bounding the LPF.

Theorem 1: For any graph $G, \sigma_{G}^{*} \geq \frac{1}{2 \beta_{G}^{*}}$.

We first show that the above theorem can be implied by the following lemma.

Lemma 2: For any graph $G, \sigma_{G} \geq \frac{1}{2 \beta_{G}^{*}}$.

Suppose that Lemma 2 holds. Then, for any nonempty subset $U$ of $V$, by applying Lemma 2 to $G[U]$, we have

$$
\sigma_{G[U]} \geq \frac{1}{2 \beta_{G[U]}^{*}} .
$$

We claim that $\beta_{G[U]}^{*} \leq \beta_{G}^{*}$. Let $H$ be an orientation of $G$ such that $\beta_{G}^{*}=\beta_{H}$. Then, $H[U]$ is also an orientation of $G[U]$. Clearly, for any node $u \in U$,

$$
N_{H[U]}^{i n}(u) \subseteq N_{H}^{i n}(u)
$$

which implies that

$$
\alpha_{H[U]}^{i n}(u) \leq \alpha_{H}^{i n}(u) \leq \beta_{H}=\beta_{G}^{*} .
$$

Therefore,

$$
\beta_{G[U]}^{*} \leq \beta_{H[U]}=\max _{u \in U} \alpha_{H[U]}^{i n}(u) \leq \beta_{G}^{*} .
$$

So our claim holds. Thus,

$$
\sigma_{G[U]} \geq \frac{1}{2 \beta_{G[U]}^{*}} \geq \frac{1}{2 \beta_{G}^{*}} .
$$

Hence

$$
\sigma_{G}^{*}=\min _{\emptyset \neq U \subseteq V} \sigma_{G[U]} \geq \frac{1}{2 \beta_{G}^{*}},
$$

and Theorem 1 follows.

In the remaining of this subsection, we prove Lemma 2 holds. We will need the following property of node-weighted digraphs proved in [12].

Lemma 3: Let $H$ be a digraph with vertex set $V$. Then for any $d \in \mathbb{R}_{+}^{V}$, there exists at least one node $u \in V$ satisfying $d\left(N_{H}^{\text {in }}(u)\right) \geq d\left(N_{H}^{\text {out }}(u)\right)$.

The next lemma gives a fundamental property of the maximal independence polytope.

Lemma 4: Suppose that $H$ is an orientation of $G$. Then for any $d \in \Omega_{G}^{*}$,

$$
1 / 2 \leq \max _{v \in V} d\left(N_{H}^{i n}[v]\right) \leq \beta_{H} .
$$

Proof: For each $I \in \mathcal{I}_{G}^{*}$, we use $\mathbf{1}^{I}$ to denote the $|V|$-dimensional indication vector of $I$. The inner product of any two $|V|$-dimensional vectors $x$ and $y$ is denoted by 
$x \circ y$. Consider any $d \in \Omega_{G}^{*}$. Then, there exist a set of pairs $\left(I_{j}, \lambda_{j}\right) \in \mathcal{I}_{G}^{*} \times \mathbb{R}^{+}$for $1 \leq j \leq k$ satisfying that

$$
\begin{aligned}
d & =\sum_{j=1}^{k} \lambda_{j} \mathbf{1}^{I_{j}} \\
\sum_{j=1}^{k} \lambda_{j} & =1
\end{aligned}
$$

Then, for any subset $U$ of $V$, we have

$$
d(U)=d \circ \mathbf{1}^{U}=\sum_{j=1}^{k} \lambda_{j} \mathbf{1}^{I_{j}} \circ \mathbf{1}^{U}=\sum_{j=1}^{k} \lambda_{j}\left|I_{j} \cap U\right| .
$$

Now, we prove the second inequality in the lemma. For each $1 \leq j \leq k$,

$$
\left|I_{j} \cap N_{H}^{i n}[v]\right| \leq \alpha_{H}^{i n}(v) \leq \beta_{H},
$$

Therefore,

$$
d\left(N_{H}^{i n}[v]\right)=\sum_{j=1}^{k} \lambda_{j}\left|I_{j} \cap N_{H}^{i n}[v]\right| \leq \sum_{j=1}^{k} \lambda_{j} \beta_{H}=\beta_{H} .
$$

Hence,

$$
\max _{u \in V} d\left(N_{H}^{i n}[v]\right) \leq \beta_{H} .
$$

Next, we prove the first inequality in the lemma. We claim that for any node $v \in V, d\left(N_{G}[v]\right) \geq 1$. Since each $I_{j}$ is a maximal independent set of $G$, we have

$$
\left|I_{j} \cap N_{G}[v]\right| \geq 1
$$

Thus,

$$
d\left(N_{G}[v]\right)=\sum_{j=1}^{k} \lambda_{j}\left|I_{j} \cap N_{G}[v]\right| \geq \sum_{j=1}^{k} \lambda_{j}=1,
$$

and hence the claim holds. By Lemma 3, there exists at least one node $u \in V$ satisfying that

$$
d\left(N_{H}^{\text {in }}(u)\right) \geq d\left(N_{H}^{\text {out }}(u)\right) .
$$

Thus,

$$
\begin{aligned}
d\left(N_{G}[u]\right) & =d(u)+d\left(N_{H}^{i n}(u)\right)+d\left(N_{H}^{\text {out }}(u)\right) \\
& \leq d(u)+2 d\left(N_{H}^{i n}(u)\right) \\
& \leq 2 d\left(N_{H}^{i n}[u]\right) .
\end{aligned}
$$

Hence,

$$
d\left(N_{H}^{i n}[u]\right) \geq d\left(N_{G}[u]\right) / 2 \geq 1 / 2 .
$$

So, the first inequality in the lemma holds.

We proceed to prove Lemma 2 using Lemma 4. Let $H$ be an orientation of $H$ satisfying that $\beta_{H}=\beta_{G}^{*}$. By definition of LPF, there exist $x$ and $y$ in $\Omega_{G}^{*}$ satisfying that $x \leq \sigma_{G} y$. Let $u$ be the node in $v$ with the largest $x\left(N_{H}^{i n}[u]\right)$. By Lemma 4,

$$
\frac{1}{2} \leq x\left(N_{H}^{i n}[u]\right) \leq \sigma_{G} y\left(N_{H}^{i n}[u]\right) \leq \sigma_{G} \beta_{H}=\sigma_{G} \beta_{G}^{*} .
$$

Hence $\sigma_{G} \geq \frac{1}{2 \beta_{G}^{*}}$. Thus, Lemma 2 holds.

\section{B. The Second Lower Bound}

In this subsection, we present another lower bound on LPF in terms of a different graph-theoretic parameter termed as the inductive local independence number. This second lower bound has a very short purely graph-theoretic proof, and yet it can imply the lower bound on LPF provided in [5] which has a queuing-theoretic proof in [5] or an alternate proof based on the duality theory of linear programming in [7]. Let $G=(V, E)$ be an undirected graph. For any node $v$, the local independence number (LIN) of $v$ in $G$ is defined to be

$$
\alpha_{G}(v)=\max \left\{|I|: I \subseteq N_{G}[v], I \in \mathcal{I}\right\} .
$$

The backward local independence number (BLIN) of $G$ is defined to be

$$
\alpha_{G}^{*}=\max _{\emptyset \neq U \subseteq V} \min _{u \in U} \alpha_{G[U]}(u) .
$$

The LPF and the BLIN of $G$ are related as stated below.

Theorem 5: For any graph $G, \sigma_{G}^{*} \geq 1 / \alpha_{G}^{*}$.

The above theorem can be implied by the following lemma.

Lemma 6: For any graph $G, \sigma_{G} \geq \frac{1}{\min _{u \in V} \alpha_{G}(u)}$.

Suppose that Lemma 6 holds. Then, for any nonempty subset $U$ of $V$, by applying Lemma 6 to $G[U]$, we have

$$
\sigma_{G[U]} \geq \frac{1}{\min _{u \in U} \alpha_{G[U]}(u)} .
$$

Hence,

$$
\begin{aligned}
\sigma_{G}^{*} & =\min _{\emptyset \neq U \subseteq V} \sigma_{G[U]} \\
& \geq \min _{\emptyset \neq U \subseteq V} \frac{1}{\min _{u \in U} \alpha_{G[U]}(u)} \\
& =\frac{1}{\max _{\emptyset \neq U \subseteq V} \min _{u \in U} \alpha_{G[U]}(u)} \\
& =\frac{1}{\alpha_{G}^{*}} .
\end{aligned}
$$

So, Theorem 5 follows.

Next, we prove Lemma 6 holds. The proof of Lemma 6 makes use of the following property of the maximal independence polytope.

Lemma 7: For any $d \in \Omega_{G}^{*}$ and any node $u \in V, 1 \leq$ $d\left(N_{G}[u]\right) \leq \alpha_{G}(u)$.

Proof: Consider any $d \in \Omega_{G}^{*}$. Then, there exist a set of pairs $\left(I_{j}, \lambda_{j}\right) \in \mathcal{I}_{G}^{*} \times \mathbb{R}^{+}$for $1 \leq j \leq k$ satisfying that

$$
\begin{aligned}
d & =\sum_{j=1}^{k} \lambda_{j} \mathbf{1}^{I_{j}}, \\
\sum_{j=1}^{k} \lambda_{j} & =1 .
\end{aligned}
$$

Then, for any $u \in V$ we have

$$
d\left(N_{G}[u]\right)=\sum_{j=1}^{k} \lambda_{j}\left|I_{j} \cap N_{G}[u]\right| .
$$


As

$$
1 \leq\left|I_{j} \cap N_{G}[u]\right| \leq \alpha_{G}(u)
$$

for each $1 \leq j \leq k$, we have

$$
d\left(N_{G}[u]\right)=\sum_{j=1}^{k} \lambda_{j}\left|I_{j} \cap N_{G}[u]\right| \geq \sum_{j=1}^{k} \lambda_{j}=1
$$

and

$d\left(N_{G}[u]\right)=\sum_{j=1}^{k} \lambda_{j}\left|I_{j} \cap N_{G}[u]\right| \leq \sum_{j=1}^{k} \lambda_{j} \alpha_{G}(u)=\alpha_{G}(u)$.

So, the lemma holds.

We proceed to prove Lemma 6 using Lemma 7. By definition of LPF, there exist $x$ and $y$ in $\Omega_{G}^{*}$ satisfying that $x \leq \sigma_{G} y$. Let $v$ be the node in $V$ with the smallest LIN. By Lemma 7,

$$
\begin{aligned}
1 & \leq x\left(N_{H}^{i n}[v]\right) \leq \sigma_{G} y\left(N_{H}^{i n}[v]\right) \\
& \leq \sigma_{G} \alpha_{G}(v)=\sigma_{G} \min _{u \in V} \alpha_{G}(u) .
\end{aligned}
$$

Hence

$$
\sigma_{G} \geq \frac{1}{\min _{u \in V} \alpha_{G}(u)} .
$$

Thus, Lemma 6 holds.

Suppose that $\prec$ is a vertex ordering of $G$. For each $v \in V$, $N_{G}^{\prec}(v)$ denotes the set of neighbors of $v$ preceding $v$ in the ordering $\prec$, and $N_{G}^{\prec}[v]$ denotes $\{v\} \cup N_{G}^{\prec}(v)$. The backward local independence number (BLIN) of a node $v$ with respect to $\prec$ is defined to be

$$
\alpha_{G}^{\prec}(v)=\max \left\{|I|: I \subseteq N_{G}^{\prec}[v], I \in \mathcal{I}\right\} .
$$

The backward local independence number (BLIN) of the ordering $\prec$ is defined to be $\max _{v \in V} \alpha_{G}^{\prec}(v)$. We show that the smallest inductive LIN of all vertex orderings in $G$ is exactly the inductive LIN $\alpha_{G}^{*}$ of $G$. For this purpose, we describe a vertex ordering, referred to as smallest-LIN-last ordering, which will be shown to achieve the smallest BLIN among all vertex orderings. This ordering is constructed iteratively as follows: Initialize $G^{\prime}$ to $G$. For $i=n$ down to 1 , let $v_{i}$ be a vertex of the smallest LIN in $G^{\prime}$ and delete $v_{i}$ from $G^{\prime}$. Then, the ordering $\left\langle v_{1}, v_{2}, \cdots, v_{n}\right\rangle$ is a smallest-LIN-last ordering.

Theorem 8: The smallest-LIN-last ordering achieves the smallest BLIN $\alpha_{G}^{*}$ among all vertex orderings.

Proof: We first show that the BLIN of any vertex ordering $\prec$ is at least $\alpha_{G}^{*}$. Let $U \subseteq V$ be such that

$$
\alpha_{G}^{*}=\min _{u \in U} \alpha_{G[U]}(u) .
$$

Let $u$ be the last vertex in the ordering $\prec$ such that $u \in U$. Then,

$$
N_{G}^{\prec}[u] \supseteq N_{G[U]}[u] .
$$

which implies

$$
\max _{v \in V} \alpha_{G}^{\prec}(v) \geq \alpha_{G}^{\prec}(u) \geq \alpha_{G[U]}(u) \geq \alpha_{G}^{*} .
$$

Next, let $\prec$ be the smallest-LIN-last ordering $\left\langle v_{1}, v_{2}, \cdots, v_{n}\right\rangle$ and we show that its BLIN is at most $\alpha_{G}^{*}$. For any $1 \leq i \leq n$, let $V_{i}=\left\{v_{1}, v_{2}, \cdots, v_{i}\right\}$. Then,

$$
N_{G}^{\prec}\left[v_{i}\right]=N_{G\left[V_{i}\right]}\left[v_{i}\right] .
$$

By the selection criteria of $v_{i}$, we have

$$
\alpha_{G}^{\prec}\left(v_{i}\right)=\alpha_{G\left[V_{i}\right]}\left(v_{i}\right)=\min _{u \in V_{i}} \alpha_{G\left[V_{i}\right]}(u) \leq \alpha_{G}^{*} .
$$

Hence,

$$
\max _{1 \leq i \leq n} \alpha_{G}^{\prec}\left(v_{i}\right) \leq \alpha_{G}^{*} .
$$

Thus, the BLIN of $\left\langle v_{1}, v_{2}, \cdots, v_{n}\right\rangle$ is at most $\alpha_{G}^{*}$.

Therefore, the smallest-LIN-last ordering achieves the smallest BLIN $\alpha_{G}^{*}$ among all vertex orderings.

Theorem 5 and Theorem 8 imply that the LPF of any graph $G$ is at least the multiplicative inverse of the BLIN of any vertex ordering of $G$.

\section{LPF OF MULTiHop WiRElESS NETWORKS}

Under either the 802.11 interference model or the protocol interference model, an instance of a network is specified by a finite planar set $V$ of nodes together with a communication radius function $r \in \mathbb{R}_{+}^{V}$ and an interference radius function $\rho \in \mathbb{R}_{+}^{V}$ satisfying that $\rho \geq r$. The communication (respectively, interference) range of a node $v \in V$ is the disk centered at $v$ of radius $r(v)$ and $\rho(v)$ respectively. The set $A$ of communication links and their conflict graph are defined as follows:

- 802.11 interference model: $A$ consists of all pairs $(u, v)$ satisfying that $u$ and $v$ are within each other's communications ranges. Two links in $A$ conflict with each other if and only if at least one link has an end lying in the interference range of some endpoint of the other link. A set $I$ of links in $A$ are independent if all links in $I$ are mutually conflict-free.

- Protocol interference model: $A$ consists of all pairs $(u, v)$ satisfying that $u$ and $v$ are within each other's communications ranges. Two links in $A$ conflict with each other if and only if the receiving end of at least one link lies in the interference range of the transmitting end of the other link. A set $I$ of links in $A$ are independent if all links in $I$ are mutually conflict-free.

Under either of the two interference models, the conflict graph $G$ of $A$ is the undirected graph on $A$ in which two links are adjacent if and only if they conflict with each other. The LPF of $G$ is referred to as the LPF of the multihop wireless network. In this section, we prove the following lower bounds on the LPF of a multihop wireless network under either the 802.11 interference model or the protocol interference model.

Theorem 9: The LPF of a multihop wireless network is at least $1 / 16$ under the 802.11 interference model, and at least

$$
\frac{1 / 2}{\left\lceil\pi / \arcsin \frac{1-\max _{v \in V} r(v) / \rho(v)}{2}\right\rceil-1}
$$

under the protocol interference model. 
By Theorem 1, it is sufficient to establish the following upper bounds on the inward LIN $\beta_{G}^{*}$ of the conflict graph $G$.

Theorem 10: $\beta_{G}^{*}$ is at most 8 under the 802.11 interference model, and at most

$$
\left\lceil\pi / \arcsin \frac{1-\max _{v \in V} r(v) / \rho(v)}{2}\right\rceil-1
$$

under the protocol interference model.

The first part of Theorem 10 under the 802.11 interference model follows from the property of an orientation $H$ of $G$ presented in [12]. For each node $v$, we use $\Gamma(v)$ to denote the set of nodes $w$ satisfying that $\rho(w) \geq \max \{\rho(v),\|v w\|\}$. Consider a link $a=(u, v) \in A$ with $\rho(u) \geq \rho(v)$. It is said to be dominated by another link $a^{\prime} \in A$ if $a^{\prime}$ has an endpoint $w$ satisfying that either $w \in \Gamma(u)$, or $w \in \Gamma(v)$ and $\|u w\|>$ $\max \{\rho(w), \rho(u)\}$. If $a$ is dominated by a link $a^{\prime}$, then $a^{\prime}$ is said to dominate $a$. The lemma below establishes the existence of the domination relationship between two conflicting links. It was proved in [12] that for any pair of conflicting links $a$ and $a^{\prime}$ in $A$, at least one of them dominates the other. So, orientation $H$ of $G$ can be constructed as follows. For any pair of conflicting links $a$ and $a^{\prime}$, if they dominate each other, we take an arbitrary orientation; otherwise, if $a$ dominates $a^{\prime}$, we take the orientation from $a$ to $a^{\prime}$; otherwise, we have that $a^{\prime}$ dominates $a$ and take the orientation from $a^{\prime}$ to $a$. It was proved in [11] that the inward LIN $\beta_{H}$ of $H$ is at most 8 . Therefore, the first part of Theorem 10 holds.

The second part of Theorem 10 under the protocol interference model follows directly from the property of an orientation $H$ of $G$ presented in [11]. For tie-breaking, all nodes are assumed to have distinct IDs. For any pair of conflicting links $a=(u, v)$ and $a^{\prime}=\left(u^{\prime}, v^{\prime}\right)$, if $v$ is within the interference range of $u^{\prime}$ and $v^{\prime}$ is within the interference range of $u$, we take the orientation from $a$ to $a^{\prime}$ in $H$ if and only if either $u$ has smaller ID than $u^{\prime}$ or $u=u^{\prime}$ but $v$ has smaller ID than $v^{\prime}$. If $v$ is within the interference range of $u^{\prime}$ but $v^{\prime}$ is outside the interference range of $u$, we take the orientation from $a^{\prime}$ to $a$ in $H$. If $v^{\prime}$ is within the interference range of $u$ but $v$ is outside the interference range of $u^{\prime}$, we take the orientation from $a$ to $a^{\prime}$ in $H$. It was proved in [11] that the inward LIN $\beta_{H}$ of $H$ is at most

$$
\left\lceil\pi / \arcsin \frac{1-\max _{v \in V} r(v) / \rho(v)}{2}\right\rceil-1 .
$$

Therefore, the second part of Theorem 10 holds.

\section{CONCLUSION}

The local pooling factor (LPF) of a multihop wireless network is exactly the throughput efficiency ratio of link scheduling strategy Longest Queue First (LQF). Under the 802.11 interference model with uniform interference radii, the LPF of a multihop wireless network was known to be at least $1 / 6$ in [5]. In this paper, we further derived lower bounds on LPFs of more general multihop wireless networks. Specifically, under the 802.11 interference model with arbitrary interference radii, the LPF is at least $1 / 16$. Under the protocol interference model, if the communication radius of each node is at most $c$ times its interference radius for some $c<1$, then the LPF is at least $1 /\left(2\left(\left\lceil\pi / \arcsin \frac{1-c}{2}\right\rceil-1\right)\right)$. These two lower bounds are applications of a general lower bound on the LPF of an arbitrary graph in terms of the inward local independence number established in this paper.

Acknowledgements: The work of P.-J. Wan, L. Wang, and Z. Wang described in this paper was supported in part by the NSF grants CNS-0831831 and CNS-0916666. The work of $\mathrm{M}$. Li described in this paper was partially supported by a grant from City University of Hong Kong under Project No. 7002611

\section{REFERENCES}

[1] A. Brzezinski, G. Zussman, and E. Modiano, Distributed Throughput Maximization in Wireless Mesh Networks via Pre-Partitioning, IEEE/ACM Trans. on Networking, Vol. 16, No. 6, pp. 1406-1419, Dec. 2008. The conference version appeared in ACM Mobicom 2006, pp. 26-37.

[2] P. Chaporkar, K. Kar, X. Luo, and S. Sarkar, Throughput and fairness guarantees through maximal scheduling in wireless networks, IEEE Transactions on Information Theory, vol. 54, no. 2, pp. 572-594, Feb. 2008.

[3] A. Dimakis and J. Walrand, Sufficient conditions for stability of longest queue first scheduling: second order properties using fluid limits, $A d$ vances in Applied Probability, vol. 38, no. 2, pp. 505-521, June 2006.

[4] C. Joo, X. Lin, and N. B. Shroff, Performance limits of greedy maximal matching in multi-hop wireless networks, in Proc. IEEE CDC'07, Dec. 2007.

[5] C. Joo, X. Lin, and N. B. Shroff, Understanding the capacity region of the greedy maximal scheduling algorithm in multi-hop wireless networks, in Proc. IEEE INFOCOM'08, April 2008.

[6] M. Leconte, J. Ni, and R. Srikant, Improved bounds on the throughput efficiency of greedy maximal scheduling in wireless networks, in Proc. ACM MOBIHOC'09, May 2009.

[7] B. Li, C. Boyaci, and Y. Xia, A refined performance characterization of longest-queue-first policy in wireless networks, in Proc. ACM MOBIHOC'09, May 2009.

[8] X. Lin and N. B. Shroff, The impact of imperfect scheduling on crosslayer rate control in wireless networks, IEEE/ACM Transactions on Networking, vol. 14, no. 2, pp. 302-315, Apr. 2006. The conference version appeared in Proceedings of IEEE INFOCOM, pages 1804-1814, 2005.

[9] G. Sharma, R. R. Mazumdar, and N. B. Shroff. On the complexity of scheduling in wireless networks, in Proc. ACM MOBIHOC'06, pages $227-238$.

[10] L. Tassiulas and A. Ephremides, Stability properties of constrained queueing systems and scheduling policies for maximum throughput in multihop radio networks, IEEE Transactions on Automatic Control, vol. 37, no. 12, pp. 1936-1948, Dec. 1992.

[11] P.-J. Wan, Multiflows in Multihop Wireless Networks, ACM MOBIHOC 2009, pp. 85-94.

[12] P.-J. Wan,C. Ma,Z. Wang, B. Xu, M. Li, and X. Jia, Weighted Wireless Link Scheduling without Information of Positions And Interference/Communication Radii, in Proc. IEEE INFOCOM 2011.

[13] X. Wu and R. Srikant, Scheduling efficiency of distributed greedy scheduling algorithms in wireless networks, in Proceedings of IEEE INFOCOM, 2006.

[14] X. Wu, R. Srikant and J. R. Perkins, Queue-Length Stability of Maximal Greedy Schedules in Wireless Networks, IEEE Transactions on Mobile Computing, June 2007, pp. 595-605.

[15] G. Zussman, A. Brzezinski, and E. Modiano, Multihop Local Pooling for Distributed Throughput Maximization in Wireless Networks, Proc. IEEE INFOCOM 2008. 